\title{
LA MANDRAGORE.
}

PAR

BERTHOLD LAUFER.

Cou Mi 周密 (1230-1320), écrivain célèbre de la fin des Song, nous a transmis une tradition fort curieuse dans ses ouvrages $K w e i$ sin tsa š 癸苹雑識 (續集上, p. 38, éd. du Pai hai) et $\check{C} i$ ya $t^{\prime} a \dot{n} 1 s a c^{\prime} a o$ 志雅堂雜鈔 (chap. 上, p. 40 b-41 a, éd. du Yüe ya $\left.t^{\prime} a \dot{n} t s^{\circ} u \dot{n} s_{s}\right) .{ }^{1}$

Le texte du Kwei sin tsa ši est ainsi conçu:

回回國之西數千里地産一物極毒全類人形 若人參之狀。其莤名之曰押不蘆。生土中深 數丈。人或誤觸之著其毒氣必死。取之法先 於四旁開大坎可容人。然後以皮條絡之。皮 條之系則繫于犬之足㿟而用杖撃逐犬。犬 逸而根拔起。犬感毒氣隨整。然後就埋土坎 中。經歲然後取出曝乾。別用他藥制之。每以 少許磨酒飲人則通身麻痺而死。雖加以刀 斧亦不知也。至三日後别以少藥挍之即活。 䓝古華陀能㓣腸滌胃以治疾者必用此藥也. 今聞御藥院中亦儲之。白廷玉聞之盧松厓。 或云。今之会官活吏䁍過盈溢被人所訟則 服百日丹者莫非用此。

' Sur cet auteur, sa vie et son cenvre, of. Pelliot, Troung Pao, 1913, p. 367-368. 
Voici le texte du Či ya t'an tsa $\check{c}^{*} a o$ :

回回國之西數千里地座一物極毒全似人形 如人參之狀。其名抑不盧。生於地中深數丈。 或從賃其皮則爌。毒之氣著人郎死。取之之 法先開大坑令四旁可容人。然後輕手以皮 條結絡之。其皮條之前則熬於大犬之足既 而用杖打犬。犬奔遠則此物拔起。大 [pour 犬] 感此氣即整。然後別埋他土中。經歲後取出 暴乾。別用藥以製治其性。以少許磨酒飲之 即通身痳牌而死。雖刀斧加之不知也。然三 日刖以少藥投之師活。蓋古者華陀能剞腸 滌臟治疾者或用此藥也。聞今御藥院中有 二枍此神藥也。白廷玉聞之盧松厓云。Le texte s'interrompt ici et n'est pas terminé.

Ni l'un ni l'autre texte ne semble être en parfait état, mais celui du Kwei sin tsa šs (A) est certainement le meilleur et le plus complet. Il est à la base de la traduction qu'on va lire, tandis que les divergences de la rédaction du Č́ ya $t^{\prime} a \dot{n}$ tsa $\check{c}^{\circ} a o$ (B) sont ajoutées en crochets.

"Quelques milliers de $l i$ à l'ouest des pays mahométans le sol produit une chose excessivement vénéneuse et pareille dans son ensemble à la figure d'un homme; en effet, elle a l'apparence du ginseng. On l'appelle ya-pu-lu (ya-pou-lou). Cette plante croit dans la terre jusqu'à une profondeur de plusieurs toises. Si un homme se heurte contre la plante par erreur, il recerra son exhalaison vénéneuse et doit mourir. [B: Quand on la blesse, son écorce brille; l'exhalaison du poison pénètre dans l'homme qui meurt aussitôt.] Voici la méthode de prendre la plante. D'abord, aux quatre côtés (autour de la racine) on crense un trou assez grand pour recevoir un homme [B: D'abord on creuse une grande fosse 
dont les quatre côtés soient assez spacieux pour recevoir un homme]. Ensuite on lie la plante au moyen d'une lanière de cuir dont l'extrémité est attachéo aux pieds d'un grand chien [B: Eusuite on lie la plante légèrement au moyen d'une lanière de cuir, dout la partie antérieure est attachée aux pieds d'un grand chien]. Arec un bâton on bat et chasse le chien qui s'enfuit en entraînant avec lui la racine, Accablé de l'exhalaison du poison, le chien périt sur le champ. Alors on ensevelit la racine dans un trou du sol [B: dans un autre sol], et au bout d'un an on l'en sort pour la sécher au soleil. Elle est mélangée avec d'autres ingrédients [B: pour dominer sa nature] et en chaque cas on en râpe un peu dans du vin qu'on donne à boire à un homme; le corps entier de celui-ci en sera paralysé, et il tombera en torpeur comme s'il était mort. Même si on lui applique des couteaux ou des haches, il ne s'en apercevra pas. Au bout de trois jours si une petite dose de médecine lui est administrée, il reviendra à la vie. C'est peut-être là le remède employé par Hwa T’o qui anciennement était capable d'ouvrir les intestins et de purger l'estomac pour guérir des malades. ${ }^{1}$ Or j'ai entendu dire qu'une provision de cette médecine [B: deux pièces, c'est une médecine divine] est conservée daus la Pharmacie. Impériale. ${ }^{2}$ C'est Pai T'iñ-yü qui l'a appris de Lu Sun-yai.

- Hwa T’o est le célèbre médecin et chirurgien qui mourut en 220 de notre ère. Cf. surtout la notice de Chavannes, BEFEO, III, 1903, p. 409. Comme M. Charannes fait remarquer d'après le Hou Han šu, l'anesthétique employé par Hwa T\%o était du chanvre infusé dans du vin qui excitait et étourdissait le patient. Done la conclusion de Cou Mi n'est pas juste. Cf. aussi C. Pétillon, Allusions littéraires, p. 380, et Toung Pao, 1898, p. 237-238; S. Julien, Chirurgie ehinoise. Substance anesthétique employée en Chine, dans le commencement du IIIe siècle de notre ère, pour paralyser momentanément la sensibitité (Comptes rendus hebdomadaires des séances de l'Académie des Sciences, XXVIII, 1849, p. 195-198). L'information est extraite de l'ouvrage médical $K u k i n$ i tui du commencement da XVIe siècle. Voir aussi Flückiger et IIanbury, Pharmacographia, p. 547. Sur les propriétés narcotiques du chanvre connaes dans l'Inde, cf. C. Joret, Les plantes dans l'antiquité, II, p. 645.

2 Cf. Bazin, Notice historique sur le collège médical de Péking, p. $24-25$ (cxtrait du .Tournal asiatique, 1856). 
Quelques uns disent: les officiers avides et les fonctionnaires oppressifs du temps présent, quand ils ont fait des exactions excessives et qu'ils sont accusés, prennent de la drogue dite drogue de cent jours; ne serait-ce pas cette plante dont ils se servent?"

Il semble que Cou Mi soit resté le seul anteur chinois à parler de la plante ya-pu-lu. Du moins, Li Ši-čen, dans son Pen ts'ao kán $m u$ (chap. 17 下, p. 13 b), ne cite-t-il que le texte du Kwei sin tsa ̌̌i à propos du ya-pu-lu; il le cite d'ailleurs assez inexactement, en supprimant le conte du chien et en ajoutant au préambule les mots 満北 mo pei, "au nord du désert Gobi." ${ }^{1}$ La dernière phrase il l'a changée ainsi: 頜官励吏罪甚者則服百日丹 皆用此也. C'est à ce texte que se rapporte la brève note de Stuart, ${ }^{2}$ qui fait remarquer qu'il u'y a pas de description de la plante, et que son identification demande de nouvelles recherches. De même, J. L. Soubeiran et Dabry de Thiersant ${ }^{3}$ ont déjà noté la plante $y a-p u-l u$ d'après le $P$ en $t s^{*} a o$ sous le titre Atropa (avec point d'interrogation), en disant: "Décrit par le Pen ts"ao comme déterminant une anesthésie suffisante pour permettre de faire des opérations. On dit que l'action s'en fait sentir pendant trois jours; il aurait été employé par le chirurgien Houa-to, pour des opérations intéressant les intestins."

Il est surprenant de voir ce que l'encyclopédie $K_{0}$ či kin yüan 格致鏡原 (chap. 69, p. 5 b) a fait du texte du Kwei sin tsa ši. Ici la plante est introduite sous le titre "herbe qui réveille de la

1 Cette addition est donnée aussi par le Yüan kien lei han (çap. 411, p. 22) dans un autre texte, de seconde main et mal digéré, concernant le ya-pu-lu. Ie $P^{e} e i$ wen čai

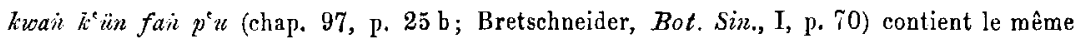
texte écourté, sans le conte du chien, mais avec l'introduction correcte $\mathbf{D}$ 回 此 方.

2 Chinese Materia Medica, P. 59; voir dejà F. P. Smith, Contributions towards the Materia Medica of China, p. 36. Smith dit sans raison que la plante vient du pays des Huns ou des Ouigours.

3 La matière médicale ches les Chinois, p. 190 (Paris, 1874). 
mort et qui rétablit la vie” 起死回生草. ${ }^{1}$ Naturellement Čou ne veut pas dire que l'homme qui prend la potion meurt réellement et ressuscite au bout de trois jours, mais seulement qu'il reste sans conscience pendant cet intervalle. S'il mourait, l'expérience de frapper le corps avec un couteau n'aurait aucun sens. Qu'il est insensible aux coups e'est la merveille; par conséquent, la vie n'est pas encore éteinte. Le conte du chien est élininé, et le document entier est abrégé ainsi: 一名抑不盧。出回回國以少

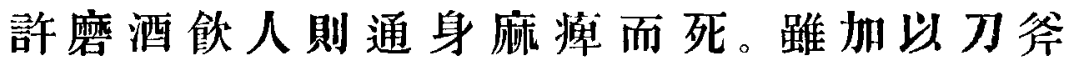
亦所不知。至三日别以少藥投之即活。御苑 中亦儲之. $\mathrm{Ce}$ texte corrompu et mutilé fut adopté par G. Schlegel, ${ }^{2}$ qui fit venir la plante de l'Arabie (au lieu des pays mahométans), l'attribua au palais impérial et induisit en erreur P. J. Veth. ${ }^{3}$

La plante décrite par Čou Mi peut être identifiée sans difficulté avec la mandragore sur la base de la transcription ya-pu-lu, laquelle correspond exactement à l'arabe-persan abruh بير ou yabruh بيزو désignation pour le fruit de cette plante. ${ }^{4}$ Elle-même s'appelle en

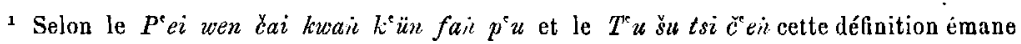
du Tien tsai ki 澳 䡛 言山.

2 Nederlandsch-chineesch Woordenboek, IV (supplément), p. 25.

3 Archives internat. d'ethnographie, VII, 1894, p. 82.

- Selon d'Herbelot (Bibliothèque orientale, I, p. 72) les Persans appellent aussi communément cette plante esterenk [astereng] et les botaniques arabes ont formé par corruption les noms d'iabroug et d'iabrouh qu'ils lui donnent, du mot persan abrou. L'origine perse du mot est plaidée aussi par Wetzstein $(Z E, 1891$, p. 891) et Veth (Archives internat. d'ethnographie, VII, 1894, p. 200) qui pour ette raison ont hasardé l'opinion que, de même, les notions magiques sur la mandragore auraient pris leur origine en Perse. C'est une hypothèse qui ne s'inspire que de considération purement philologique sur les mots; les preuves historiques font défaut: Il n'y a pas de texte iranien de date ancicnne à ce sujet. Pour la première fois la mandragore est mentionnée dans la littérature perse par Abu Mansur, qui autour de l'an 975 écrivit son Livre des Principes Pharmacologiques (traduction d'Achundow, p. 148). Baber écrit dans ses Mémoires que la mandragore se trouve dans les montagnes du Fergana (A. S. Beveridge, Memoirs of Bābur, p. 11). Il est difficile de se ranger à l'avis de Wetzstein que l'arabe yabrïh serait issu du persan abrewi. Voir aussi Horn, Grundr. iran. Plil., I, 2, p. 73 . 
arabe toffāh-el-jenn تخف ("la pomme des esprits") ou siräj elkotrob سؤج القطرب ("la lampe des lutins"), aussi la'ba et beid el-jinn ("œufs des esprits"). 1 En Araméen le fruit est nommé

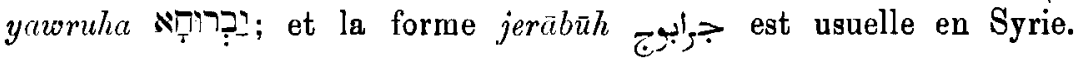
Ce nom sémitique paraît être d'une date relativement ancienne; du-moins trouvons-nous dans Dioscoride un terme dit égyptien de

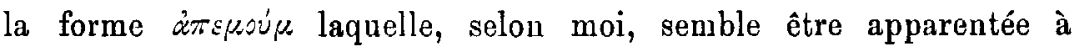

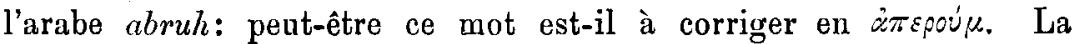
mandragore (ou mandegloire par étymologie populaire) ${ }^{2}$ forme un genre de la famille des solanées à la racine fusiforme et souvent bifurquée, aux feuilles radicales d'un vert sombre, aux fleurs purpurines et dont les fruits rouges, semblables à une petite pomme, exhalent une odeur agréable. ${ }^{3}$ Les propriétés narcotiques de cette

1 L. Leclerc, Traite des simples, II, p. 246; III, p. 240; d'Herbelot, Bibliothèque orientale, III, p. 524. Les Arabes ont emprunté aux Grecs aussi le mot mandragoras dans la forme mandaghuru (Leclerc, III, p. 34l).

2 Francisque-Michel, Recherches sur le commerce, la fabrication et l'usage des étoffes de soie, II, p. 76, Paris, 1854) a fait cette observation: "Au XVe siècle, ils [nos ancêtres] employaient la soie à conserver certaines amulettes, dont un célèbre prédicateur de l'époque brûla un grand nombre, ce qui valait mieux assurément que de brûler les gens qui y avaient foi. On les appelait madagoires, par une altération du mot mandragores. 'A ujourd'huy, ajoute l'auteur du Journal du roy Charles VII, le vulgaire les appelle mandegloires, que maintes sottes gens gardoient en lieux de repos, et avoient si grande foy en celle ordure, qu'ils sroyoient fermement que tant comme ils l'avoient (mais qu'il fust bien nettement en beaux drapeaux de soye ou de lin envelopé), jamais jour de lenr vie ne seroient pauvres.' Dans le dialogue de Mathurine et du jeune du Perron, celui-ci lui dit: 'As-tu point aidé a souftler le feu lent sous la coque d'auf où est le germe, la soye cramoisie, et cela de quoy les magieiens faisoient leur pâque avec la petite mandragore?' (Confession catholique du sieur de Sancy, liv. II, ch. ler)." Aussi l'expression main de gorre étuit en usage populaire.

3 C. Joret, Les plantes dans l'antiquité et au moyen age, I, p. 498. - "Ia Mandragora officinarum est connue sous le nom de Manaragore femelle. Elle est très commune dans le midi de la France, on la rencontre en abondance sur les rivages de la Calabre, de la Sicile, de lî̀le de Crète, de la Cilicie, de l'Afrique, de l'Espagne; elle se plaît dans les lienx ombragés, sur les bords des rivières, à l'entrée des cavernes. Elle fleurit en automne, quelquefois aussi au printemps. Sa racine est grosse, noirâtre extérieurement, blanche à l'intérieur, charnue; ses feuilles sont grandes, les plus extérieures obtuses, les plus intérieures aiguës; leur couleur est un vert bleuâtre, luisant en dessus, terne en dessous. Le pétiole est long; les hampes florales sont longues, rougeâtres, et un peu pentagonales. 
plante étaient connues anciennement, et elle était douée de vertus magiques, aphrodisiaques et prolifiques. La racine prend souvent des formes singulières, rappelant plus ou moins le corps de l'homme. Le nom est dérivé du grec pavjpxyopxe, mot dont l'étymologie est encore inconnue. D'après Littré, ce paraît être un nom d'homme appliqué à une plante, et contenir $\mu \dot{x} y \delta \rho o s$ ou $\mu x \dot{x} \delta \rho x$, nom d'uue divinité locale de l'Asie Mineure. L'origine orientale de plusieurs croyances attachées à cette plante, comme nous verrons, paraît certaine. Assurément, le nom n'a rien à voir avec le persan mardum-

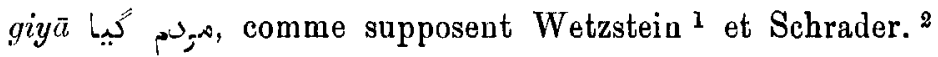

Ce n'est pas le but de cette notice de retracer toutes les croyances touchant les vertus de la mandragore et accumulées pendant beaucoup de siècles. Un tel travail a été maintes fois tenté, mais, à vrai dire, aucun essai de ce geure n'est tout à fait satisfaisant ou complet daus l'emploi des sources. ${ }^{3}$ Une œurre d'ensemble et

La fleur se compose d'un calice à cinq divisions aiguës et lanceolées, d'une corolle trois fois plus longue que le calice, de couleur violette et découpée en cinq lobes oblongs, obovés" (A. Milne Fdwards, De la fanille solanacács, p. 56, Paris, 1864).

1 L. c.

2 Reallexilon, p. 36. Line nouvelle hypothèse sur l'aftinité du nom grec se trouve à la conclusion de cet article.

${ }^{3}$ Il y a trois monographies que je n'ai pas en l'occasion de voir, J. Schmidel, Dissertatio de mandragora (Lipsiae, 1671); Granier, Dissertation lotanique et historique sur la mandragore (Paris, 1788); et Bartolomi, Commentarii de mandragoris (Bologna, 1835). Ce sont les traités suivants qui me sont connus: F. v. Luschan a illustré six racines de mandragore de l'Orient proche sculptées en figures humaines $(Z E, X X I I I, 1891$, p. 726-728); sa brève notice est accompaguée de notes explicatives par Ascherson et Beyer (p. 729-746) et de notes additionnelles par Wetzstein (p. 890-892). - W. Hertz, Sage vom Giftmädchen, traite de la mandragore dans un appendice ( $A B A W, 1893$, p. 164-166). P. J. Veth, De alruin en de heggerank (Archives internationales d'ethnographie, VII, 1894, p. 81-88) et Do mandragora (ibid., p. 199-205). - C. B. Randolph, The Mandsagora of the Ancients in Folk-Lore and Medicine (Proceedings American Academy of Arts and Sciences, XL, Boston, 1905, p. 487-537). - E. O. v. Lippmann, Alraun und schwarzer IIund, dans ses Abhandlungen, I, 1906, p. 190-204. - Comme on suppose qu'il s'agit

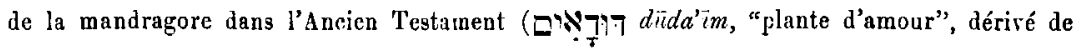
$d \bar{u} d$, "aimer"; Gen., XXX, 14-16, et Cant., VII, 14), on trouve des articles à ce snjet dans les nombreux dictionnaires bibliques; le meilleur que j'aie vu est celui de $\mathrm{E}$. Levesque 
de critique reste à faire. Les notes suivantes ne doivent être regardées que comme un commentaire du texte de Cou $\mathrm{Mi}$; toutefois rien d'important n'y est omis.

au Dictionnaire de la Bible par F. Vigouroux (IV, col. 653-655). Il ne faut pas oublier que cette interprétation du terme hébreux repose sur une hypothèse, d'ailleurs fort vrai-

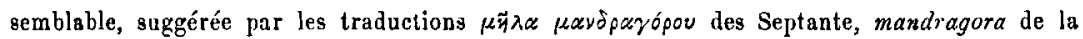
Vulgate, et yābruḩin du Targum d'Onkelos et du syriaque; en outre, la plante est répandue en Palestine. - Le savant japonais Kumagusı Minakata (Nature, LI, 1895, p. 608; et LIV, 1896, p. 343-344; ef. Teoung Pao, 1895, p. 342) a contribué deux brèves notices à ce sujet en se servant de sources chinoises, mais sans méthode et critique. Je ne veux pas entrer daus une critique détaillée de ce travail, mais je voudrais remarquer seulement

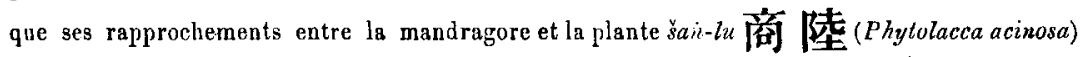
ne sont que des parallèles psychologiques, mais non historiques (voir infra). Minakata a aussi donné une traduction du conte de Č́ou Mi avec quelques contre-sens sans consulter le texte meilleur du $K w e i$ sin $t s a$ ši, et a fait allasion à Josèphe par des sources de seconde main. Je ne dois rien à cette étude; en effet, j’ai trouvé tous les textes indépendamment, et mon travail était achevé quand par hasard l'artjele de Minakata est tombé dans mes mains. - Niccolò Macchiavelli (1469-1527) est l'auteur d'une comédie, d'abord intitulée Comedia di Callimaco et di Lucrezia (lre édition, s.l.n.d.), puis Mandragola (1524, etc.; éd. sous mes yeux, Roma, 1688), en cinq actes, en prose, précédéc d'un prologue; c'est une satire sur la croyance à la vertu de la mandragore polir féconder une femme. Callimaco dit a Mèsser Nicia (p. 63): "Voi havete a intendere questo, che nõ è cosa piu certa a ingravidare d'une potione fntta di Mandragola, questa è una cosa esperimẽtata da me due para di volte, et trovata sempre vera: e se non era questo, la Reina di Francia sarebbe sterile, e infinite altre principesse di quello stato." La comédie de Machiavel a fourni à J. de la Fontaine le sujet d'un conte rimé qui est intitulé "La Mandragore, nouvelle tirée de Machiavel" (Ceuvres de $J$. de la Fontaine par H. Regnier, tome V, 1889, p. 22, avec une introduction intéressante de l'éditeur).

"Cette recette est une médecine

Faite du jus de certaine racine,

Ayant pour nom mandragore; et ce jus

Pris par la femme opère beaucoup plus

Que ne fit onc nulle ombre monacale

I'aucun couvent de jeunes frères plein".

La Mandragola a été imitée par J.-B. Rousseau dans sa comédie la Mandragore, également en cinq actes, en prose, "tirée, dit le titre, de l'italien de Machiavel". Andrea Calmo écrivit la Potione, comedia facetissima et dilettevole, en quatre actes et un prologue, imitation de la Mandragola, écrite dans les dialectes vénitien, bergamasque, italo-grec, etc. (Venise, 1552, réimprimée en 1560, 1561, et 1600). Il y a une nouvelle de Charles Nodier, intitulée la Fée aux miettes (1832), dont le héros, pour posséder sa maîtresse, doit trouver "la mandragore qui chante". Une nouvelle allemande Mandragora, d'ailleurs assez faible, par de la Motte Fonqué, a para en 1827. 
L'historiette du ehien déracinant la plante ne se trouve ni dans Pline ni dans Dioscoride qui l'un et l'autre ont écrit sur la mandragore. La version la plus ancienne que nons connaissions est due à Flavius Josèphe (37-93) qui dans son curre De bello judaico (VII, 6, $§ 3$ ), écrit entre les années 75 et 79 , s'exprime ainsi: ${ }^{1}$ "Or dans ce palais croissait une espèce de rue ${ }^{2}$ qui mérite notre admiration à cause de ses dimensions, car elle était aussi large qu'un figuier en ce qui concerne la hauteur et l'épaisseur; et, suivant une tradition, elle avait duré depuis le temps d'Hérode, et probablement elle aurait continué beaucoup plus longtemps si elle n'avait pas été tranchée par les Juifs qui occupaient la place plus tard. Et dans la ravine qui environne la cité [Machaerus] au côté du nord, il y $a$ une certaine place nommée Baaras et produisant une racine da même nom. Sa couleur est semblable à celle du feu, et vers le soir, elle émet un rayon comme un éclair. Elle n'est pas prise aisément par ceux qui s'approchent d'elle et désirent l'enlever, mais elle se

- retire de leurs mains et n'est pas stationnaire jusqu'à ce que l'urine ou le sang menstrual d'une femme soient versés au-dessus d'elle. Même alors ceux qui la touchent rencontreront une mort certaine s'ils ne portent suspendue à la main une racine de la même espèce. Il y a aussi une autre méthode de l'ôter sans risque, et la voici. Les gens creusent le sol autour de la plante jusqu'à ce que la partie cachée de la racine devienne fort petite. Alors ils y lient un chien, et quand le chien suirra la personne qui l'a lié la racine est arrachée sans difficulté; mais le chien expire infailliblement, comme s'il était une victime au lieu de l'homme qui devait preudre la plante. Après cela, personne n'a besoin de craindre de la prendre dans ses mains. Cependant, après tous ces dangers qu'on court à

- Flavii Josephi opera graece et latine ed. G. Dindorfius, II, p. 316 (Parisiis, 1865)

2 Une herbe de la famille Rutaceae, mentionnée par Luc (XI, 42). Plusieurs espèces sauvages croissent en Palestine, tandis qu'une espèce, Ruta graveolens, est cultivée. 
l'obtenir, elle n'est recherchée qu'en considération d'une seule propriété qu'elle a, à savoir que, apportée à des malades, elle chassera vite les démons (qui ne sont autres que les esprits des méchants) qui entrent dans les hommes vivants et les tuent, s'ils ne peurent pas obtenir de secours contre eux." I

La cité de Baaras était située en Syrie, sur le bord oriental de la mer Morte. Josèphe ne donne pas le nom de la plante, mais il n'y a pas de doute qu'il ait envisagé la mandragore qui existe en Palestine. Le motif de la racine arrachée par un chien paraît être d'origine orientale, et ensuite fut adopté par l'bellénisme lequel a absorbé tant d'idées orientales. ${ }^{2}$

Un conte semblable est raconté par F́lien (Hist. an. XIV, 27)

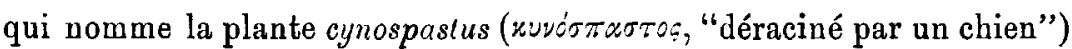

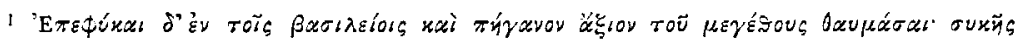

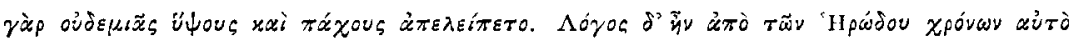

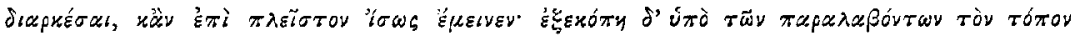

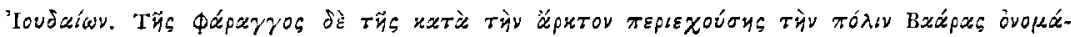

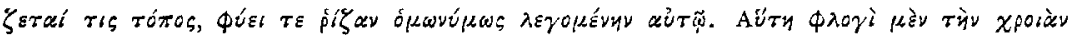

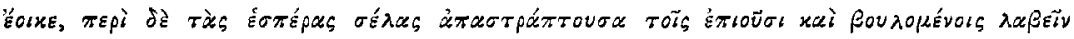

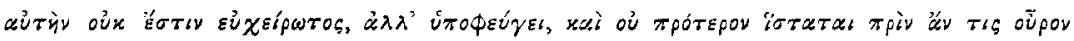

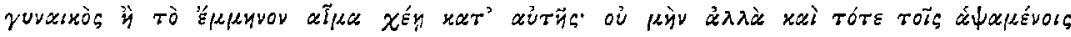

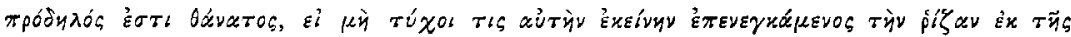

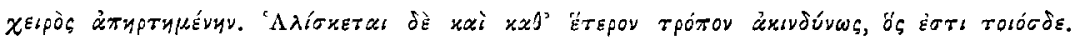

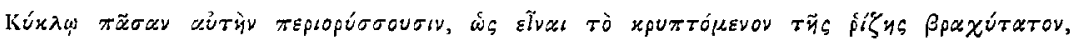

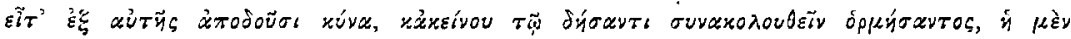

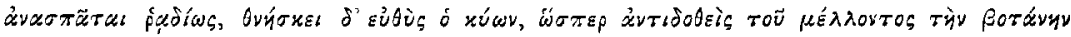

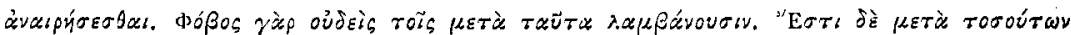

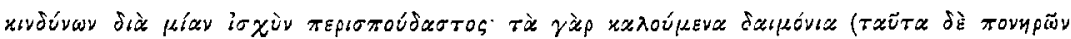

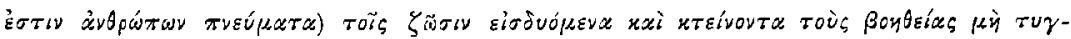

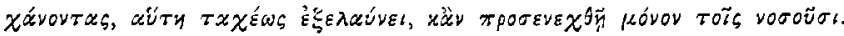

3 Je m'abstiens d'aborder le problème botanique. Dans la plupart des cas il est impossible d'insister sur uue identification trop spécifique. Mandragora officinalis, Atropa mandragora, ou même Atropa beliadonna ont été proposées comme les plantes comprises par les anciens à ce titre. Je ne crois pas cependant qu'une seule espèce y corresponde, car les mêmes idées pouvaient passer d'uue plante à l'autre. On sait que la mandragore n'a jamais pénétré au-delà des Alpes sauf dans le midi de la France; néanmoins on a réussi à en trouver des substituts dans l'Europe centrale et septentrionale. 


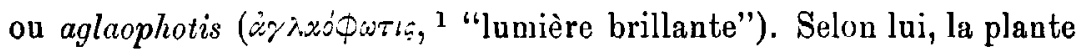
est cachée au-dessous parmi les autres herbes pendant le jour, tandis que de nuit elle devient visible et luisante comme une étoile, car elle rayonne et ressemble à du feu ( тupi $=$ 熫 ou 是). Par conséquent les gens attachent un signe distinctif à la racine et s'éloignent. Sans cette précaution, ils ne peuvent pas se souvenir au jour de la couleur ni de la figure de la plante. Mais ils n'ont pas coutume d'extraire ce végétal euxmêmes, car on dit que celui qui l'a touché par ignorance de sa nature meurt quelque temps après. On conduit donc un chien jeune et robuste qui n'a point reçu de nourriture pendant quelques jours et qui a une faim violente; on le lie à une corde forte aussi loin que possible, et l'on fait un nœud difficile a dénouer, autour du bas de la tige de l'aglaophotis. Un repas opulent de viande rôtie, d'une odeur suave, est présenté au chien qui, poussé par la faim et attiré forcément par la bonne odeur de la viande, arrache la plante arec la racine. Quand le soleil regarde la racine, le chien mourra aussitôt. Les gens l'enserelissent à la même place, et ayant rempli quelques cérémonies mystérieuses en honorant le cadarre du chien, parce qu'il a laissé sa vie pour eux, ils osent toucher le végétal et le portent chez eux. Ils l'emploient pour beaucoup de choses utiles, et à ce qu'on dit, ceux qui souffrent de l'épilepsie en sont guéris; elle est bonne aussi pour la maladie des yeux.

Le conte d'Élien, sans doute un peu loquace, n'est pas localisé, et est un peu exagéré: il n'y a guère de lieu pour le repas, à moins que ce ne fût un acte de charité. Le texte d'Élien qui vécut à Praeneste en Italie sans jamais quitter ce pays démontre que le conte fit sa migration de l'Orient en Italie.

Pline, afin d'illustrer les mensonges des magiciens anciens, dit que dans sa jeunesse le grammairien Apion lui parla de la plante

' Cf. Pline XXIV, 102. 
cynocephalia ("tête de chien"), connue en Egypte sous le nom d'osiritis, utile pour la divination et préservatif contre tous les maurais effets de la magie; mais si quelqu'un l'arrache da sol dans sa totalité, il mourra aussitôt. ${ }^{1}$ C'est la même superstition que nous avons trouvé dans Josèphe et Élien, et ici même l'Orient (l'Egypte et les magiciens) paraît en preudre la responsabilité. Si le nom cynocephalia, qui avant tout se rapporte à la forme de la plante, permet d'établir un rapprochement avec le chien de Josèphe et d'Élien, e'est ce que je n'ose décider. ${ }^{2}$

La légende occidentale reproduite par Cou Mi présuppose éridemment une version d'origine islamique qui doit s'être répandue en Chine à l'époque des Song. En consultant la vaste compilation d'Ibn al-Baițār dans l'excellente traduction de L. Leclerc, ${ }^{3}$ nous n'en trouvons pas de trace. Malheureusement, Leclere a cru bon d'éliminer quelque chose de cet article, car il ajoute: "Quelques passages de ce chapitre, qui tranche par son caractère sur le ton général de l'ourrage d'Ibn al-Baițīir, nous ont paru devoir être supprimés." J'ai done recouru à la traduction de Sontheimer, laquelle, comme on sait, est bien inférieure à celle de Leclere à tous égards, et j'attends, d'ailleurs, la confirmation de ce texte par un arabisant. Selon Sontheimer, ${ }^{4}$ Ibn al-Baițār mentionnerait le procédé avec le chien et ajouterait que lui-même en a été témoin, mais qu'il a trouvé faux que le chien y perde sa vie.

1 Quaerat aliquis, quae sint mentiti vetcres Magi, cum adulescentibus nobis visus Apion grammaticae artis prodiderit cynocephalian herbam, quae in Aegypto vocaretur osiritis, divinam et contra omnia veneficia, sed si tota erueretur, statim eum, qui eruisset, mori (XXX, 6, 18).

2 Dans un autre passage de Pline (VIII, 27, 101) les fruits de la mandragore sont nuisibles aux ours qui lèchent des fourmis comme antidote (Ursi cum mandragorae mala ustavere, formicas lambunt); cf. Solinus (XXVI, 8): Cum gustavere mandragorae mala, moriuntur: sed eunt obviam, ne malum in perniciem convalescat et formicas vorant ad uperandam sanitatem.

3 Traité des simples, II, p. 24,6-248.

- II, p. 14. 
Dans la traduction de Leclerc l'auteur arabe fait dire à Hermès à propos de l'acquisition de la plante qu'on prétend que son extraction est difficile par la raison qu'il faut connaitre le temps favorable à l'opération. ${ }^{1}$ D'autre part, d'Herbelot ${ }^{2}$ a rérélé une version qui s'approche assez nettement du texte de l'écrivain chinois. "Luthf-Allah dit qu'il g a du danger d'arracher, ou de couper cette plante, et que pour éviter ce danger, quand on veut la tirer de terre, il faut attacher à sa tige un chien que l'on bat ensuite, afin que faisant des efforts pour s'enfuir, il la déracine." Voilà le trait de battre le chien, étranger à Josèphe et Élien, mais admis dans la version chinoise. Cependant un parallèle arabe plus complet et plus exact reste à chercher. D'ailleurs, autant que je sache, il n'y a pas beaucoup d'originalité daus les notices des Arabes sur la mandragore. Par exemple, tout ce qui est rapporté par Qazwīnì à ce sujet, comme l'a reconnu aussi G. Jacob, ${ }^{3}$ n'est que l'écho des traditions hellénistiques. Qazwīñ̄ a copié Avicenne (980-1037), et Avicenne a été répété par les historiographes européens des croisades et d'autres écrivains médiévaux. Enfin, les auteurs byzantins comme Théophane et Kedrenos ne font que reproduire les traditions des anciens.

Pour ce qui est des propriétés Iumineuses de la plante, nous les avons vues accentuées par Josèphe et Élien. Le chérif el-Edrisy fait remarquer: "On donne à cette plante le nom de siräj el-kotrob, parce que le kotrob est cette petite bête qui luit la nuit comme du feu. Cette plante est bien connue en Syrie où elle croît surtout non loin du littoral. La partie interne de l'écorce de sa tige luit la nuit, tant qu'elle reste humide, au point qu'on la croirait embrasée. Une fois desséchée, elle perd cette propriété. Si on la met

1 L. Leclerc, Traité des simples, 11, p. 247.

- Bibliothique orientale, 1, p. 72.

- Studien in arabischen Geographen, p. 165. 
dans un linge mouillé, l'humidité lui rend cette lueur qu'elle perd en se desséchant." 1

La forme anthropomorphique de la plante (plus correctement de la racine) sur laquelle insiste Cou Mi n'est pas relevée par les auteurs classiques. Dioscoride décrit la racine ${ }^{2}$ sans meutionner cette qualité. Cependant, nous apprenons par une citation du Codex neapolitanus de Dioscoride que la racine de la mandragore

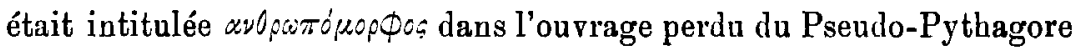
sur les effets des plantes. De même, Columella (De re rustica $X, 19,20$ ) en parle au terme planta semihominis.

Hermès est cité par Ibri al-Baițār comme disant: "La racine souterraine de cette plante a la forme d'une idole debout, avec des pieds et des mains et tous les organes de l'homme. Sa tige et ses feuilles, issues de la tête de cette idole, apparaissent à l'extérieur, et les feuilles ressemblent à celles de la ronce. Elle s'attache anssi aux plantes qui l'avoisinent et s'étale par-dessus." 8

La qualité soporifique de la plante est signalée par Aristote (De somno et vigilia), Théophraste (Hist. plant. IX, 9, 1) et Xénophon (Symp. II, 24). Dioscoride (IV, 76) dit qu'elle fournit un suc endormant, étourdissant ou même mortel, employé par les médecins comme anesthésique sous forme de vin pour les opérations chirurgicales at qu'elle s'atteste comme aphrodisiaque efficace.

Lucien fait deux allusions à cet effet du remède: "tu dors, comme assoupi par de la mandragore"; et Démosthène réveille, malgré eux, ses concitoyens assoupis comme s'ils avaient bu de la mandragore. ${ }^{4}$

1 L. Leclerc, Traité des simples, II, p. 247.

2 Jes racines sont très longues, au nombre de deux ou trois, intriquées l'une dans l'autre, noires en dehors, blanches en dedans et recouvertes d'une écorce épaisse (L. Iseclere, Traite des simples, III, p. 419); mais Pline et Dioscoride sont d'accord pour rapporter que la plante se présente sous deux sexes, mâle et femelle.

3 I. Leclerc, Traité des simples, II, p. 247.

4 E. Talbot, Ouvres complètes de Lucien de Samosate, I, p. 31; II, p. 474 (Tim. 2, Dem. Enc., 36). 
Pline aussi en signale la force soporifique, mais la dose devait être réglée proportionnellement à la vigueur du malade. De plus, on la burait contre des morsures de serpents et pour assurer l'insensibilité avant des opérations; l'odeur en suffisait à quelqu'uns pour produire le sommeil. ${ }^{1}$ Théosphraste ${ }^{\&}$ dit qu'elle induit en sommeil, mais que donnée en plus grande quantité, elle est mortelle (oi j'javwrixoi

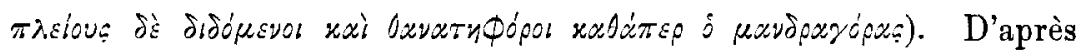
Celsus (III, 18), les anciens avaient l'habitude de mettre le fruit de la plante sous leurs oreillers pour hâter le sommeil.

Hermès, cité par Ibn al-Baițār, dit que c'est une plante bénie entre toutes et qu'elle est utile contre toutes les maladies qui affligent l'homme par le fait des génies, des démons (cf. Josèphe) et de Satan. Elle est salutaire aussi contre les graves affections internes, telles que la paralysie, le tic nerveux, l'épilepsie, l'éléphantiasis, l'aliénation mentale, les convulsions et la perte de la mémoire. ${ }^{3}$

Le vin mentionué par Čou Mi et Dioscoride, dans lequel on a fait infuser des racines de mandragores s'appelait mandragorite (Littré). En italien c'est mandragolato. L'usage de ce terme remonte jusqu'à Dioscoride $\left(\mathrm{V}, 81: \delta \mu \alpha \nu \delta\right.$ paropituc oivoc). Théophraste ${ }^{4}$ a déjà fait observer que la racine est administrée dans du vin ou du vinaigre

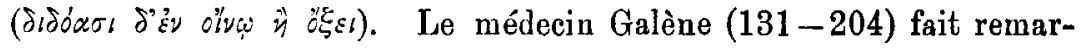
quer que l'extrait de mandragore, aussi bien que le vin qu'il servait à préparer, étaient chaque an apportés de Crète à Rome. Ajoutons le texte de l'érêque Isidore (Isidorus Hispalensis, ca. $570-636)$, inséré dans ses Originum sive etymologiarum libri $X X$ (XVII, 9): "Mandragora dicta, quod habeat mala suaveolentia in

1 Vis somnifica pro viribus bibentium; media potio cyathi unius. Bibitur et contra serpentes et ante sectiones punctionesque, ne sentiantur; ob haec satis est aliquis somnum odore quaesisse (XXV, 94, $\$ 150)$.

- De causis plantarum, VI, 5.

- L. Leclerc, Traité des simples, II, p. 246.

- Historia plantarum, IX, 9, 1. 
magnitudinem mali Martiani; unde et eam Latini malum terrae rocant. Hanc poetae $\dot{\alpha} \nu 0 \omega \pi \hat{\beta}_{\mu}$ op $\phi$ ov appellant, quod habeat radicem

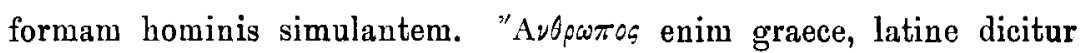
homo. Cuius cortex vino mixtus ad bibendum datur iis quorum corpus propter curam secandum est, ut soporati dolorem non sentiant. Huius species duae: foemina, foliis lactucae similibus, mala generans in similitudinem prunorum; masculus vero folia betae similia habet." I

Nous devons tourner maintenant vers une autre idée attachée à la mandragore, qui ne se troure pas chez Cou $\mathrm{Mi}$, mais qui se manifeste dans un autre groupe de traditions chinoises. Maimonides (1135-1204) dit à propos du livre L'Agriculture des Nabatéens ${ }^{2}$ que Adam dans son livre fit mention d'un arbre dans l'Inde, les branches duquel rampent comme un serpent, quand on les jette sur terre; et, de même, d'un autre arbre, la racine duquel a la forme d'un homme et une haute voix et prononce des paroles intelligibles. $^{8}$

Nous lisons dans la matière médicale d'Ibn-al-Baițār (1197-1248) sur la plante luf لوف (Arum dracunculus): "Il y en a trois espèces. L'une s'appelle en grec dracontion, ce qui veut dire arum serpentaire, x.̣̂, à cause que sa tige tachetée ressemble à une peau de serpent. C'est l'arum loug, لوف كبير Nos compatriotes en Espagne Iui donnent le nom de gargantīa غرغنتيه. D'autres l'appellent sarrākha $2 \dot{>}^{\prime}$, , parce qu'ils prétendent qu'elle jette un cri, sarkha, que l'on entend le jour du Mihrijān, c'est-à-dire

1 L'idée que la mandragore hâte la propagation émane pour la première fois da Physiologus (chap. XIX), où la plante est localisée près du paradis, étant cherchée et mangée par les éléphants avant de s'accoupler. Je ne poursuis pas cette piste ici, parce que cette notion ne joue pas de rôle dans Ia tradition cbinoise.

2 Cf. E. Renan, An Essay on the Age and Antiquity of the Book of Nabathaan Agriculture (London, 1862); A. v. Gutschmid, $Z D M G, X V$, p. 1, et Nöldeke, ibid., XXIX, p. 445. On sait que ce livre (Falātıa nabatīya) qui prétend d'être une traduction arabe d'une ancienne source nabatéenne est une forgerie du dixième siècle.

3 D. Chwolson, Ssabier, II, p. 458. 
le jour de la Pentecôte, et, de plus, que celui qui l'entend mourra dans l'année." I La même observation est aussi faite par Ibn el'Awwām de Séville, qui écrivit dans la première moitié du VI ${ }^{\theta}$ siècle de l'hégire le Kitāb el-falāha (Lirre de l'agriculture). ${ }^{2}$ L'analogie de ce cas avec la mandragore est frappante, et il s'agirait de savoir si le trait de la plante qui pousse un cri et cause la mort d'un homme était à l'origine propre à l'arum, c'est-à-dire, appartenait à un autre cycle de traditions, et a passé de là à la mandragore, ou inversement. En tout cas cette notion légendaire paraît bien être d'origine orientale. Autant que je sache, Maimonides ou plutôt l'œuvre apocryphe qu'il cite présente la source la plus aucienne qui contienne la combinaison de cette attribution avec la mandragore. Dès ce temps-là ce motif ne tarda pas d'être vulgarisé: le cri poussé par la racine de la mandragore au moment qu'elle est arrachée au sol devient fatal à l'auditeur. Le plus fameux passage de ce genre se trouve dans Shakespeare, Romeo and Juliet (IV. 3, 47):

And shrieks, like mandrake's torn out of the earth, That living mortals, hearing them, run mad.

Dans King Henry VI (II. 3, 2) Suffolk dit à la reine:

Would curses kill; as doth the mandrake's groan. ${ }^{3}$

1 L. Leclerc, T'raité des simples, III, p. 248.

2 C. Huart, Littérature arabe, p. 313. L'ouvrage d'Jbn el- Awwam a été traduit en français par J.-J. Clément-Mullet (Ibn al Awwam, livre de l'agriculture, 2 vols., Paris, 1864-1867). Malheureusement je a'ai pas accès à cette traduction; j'ai tiré le fait en question de I. Jöw, Aramäische Pfanzennamen, p. 239.

3 Dans plusieurs autres passages, Shakespeare fait allusion à la mandragore.

Not Poppy, nor Mandragora,

Nor all the drowsie Syrrups of the world

Shall ever medicine thee to that sweet sleep

Which thou owedst yesterday.

Othello, III. 3, 330.

Give me to drink mandragora...

That I might sleep out this great gap of time.

Anthony and Cleopatra, I. 5.

Dans King Henry IV (II. 1, 2), Falstaff appelle son petit page "whoreson mandrake"; le 
Mais hâtons-nous d'ajouter que cette tradition est strictement médiévale. C'est par inadvertence que G. E. Post ${ }^{1}$ fait observer, "The ancients also believed that this root gave a demoniacal shriek as it was pulled up." Il n'en est rien: rien de pareil dans aucun document de l'antiquité.

Cette idée bizarre, d'où vient-elle? Nous avons vu que Čou Mi compara la mandragore avec le ginseng (Panax ginseng), fameuse panacée de sa patrie. D'autre part, le nouveau dictionnaire anglais d'Oxford régistre le terme "Chinese mandragoras" au sens de ginseng, et le dictionnaire persan-anglais de Steingass donne cette définition de l'expression mardum-giya said to resemble a man and woman, and to which many wonderful effects are attributed; mandrake, colocynth." De cette manière, le mot persan désigne la mandragore aussi bien que le giuseng d'origine chinoise. C'était le P. Martini (1655) qui rapprocha le dernier à la maudragore: "Je ne sçaurois mieux representer cette racine qu'en disant qu'elle est presque semblable à nostre Mandragore; hormis que celle-là est un peu plus petite quoyqu'elle soit de quelcune de ses especes. Pour moy je ne doute point du tout, qu'elle n'ayt ces mesmes qualités et une pareille vertu; puisqu'elle luy ressemble si fort et qu'elles ont toutes deux la mesme figure" [suit une assez longue description de la racine et de ses propriétés]. ${ }^{2}$ De même

juge Shallow recevait dans sa jeunesse le sobriquet "mandrake" ("when he was naked, he was...like a forked radish with a head fantastically carved upon it with a knife"; ibid., III. 2). Enfin le passage dans Macbeth (I. 3, 84)

Or have we eaten of the insane root

That takes the reason prisoner?

paraît contenir une allusion à la mandragore.

1 Dans le Dictionary of the Bible de J. Hastings, III, p. 234.

'A. Kircher, La Chine illustrée, p. 241 (Amsterdam, 1670). On voit ainsi que le ginseng était connu en Europe au XVII ${ }^{e}$ siècle. Je ne m'arrête pas à cette matière sur laquelle tant a été écrit. Il suffit de renvoyer le lecteur à Bretschneider, Butanicon sinicum, $3^{2}$ partie, no. 3; Du Halde, Description de l'empire de la Chine, II, p. 150 (ce mémoire est dû au P. Jartoux); Némoires concernant les Chinois, II, p. 428; et voir la bibliographie 
que la mandragore, le ginseng est anthropomorphisé et doué de langage par les Chinois. L'ourrage ancien Pie lu 别錄 dit que sa racine est comme la figure de l'homme et a des qualités divines (根如人形者有神); et le $W u$ pu pen ts ao 䞨萻本草， écrit au troisième siècle, attribue à la racine des mains, des pieds et des yeux, tout comme chez l'homme, et la range parmi les choses spirituelles (根有手足而目如人者神). ${ }^{1}$ Ensuite le ginseng est capable de crier. Le document le plus ancien à cet égard qui me soit connu est contenu dans les Annales de la dynastie Soui, où nous lisons: "Au temps de Kao Tsu (ou Wen Ti, 590-604)

dans H. Cordier, Billiotheca sinica, col. 2969, 3055-6. - L'observation du P. Martini fut relevée par J. F. Lafitau (Mémoire presenté à son altesse royale Monseigneur le Duc d'Orleans, regent du royaume de France; concernant la précieuse plante du ginseng de Tartarie, découverte en Canada, 88 p., petit $8^{\circ}$, Paris, chez J. Monge', 1718), missionnaire Jésuite parmi les Iroquois, qui, après avoir la le mémoire de Jartoux sur le ginseng chinois, découvrit une semblable espèce au Canada. Il dit (p. 71): "Quand j'eus découvert le ginseng, il me vint en pensée que ce pouvoit être une espece de mandragore. J'eus le plaisir de voir que je m'étois rencontré sur cela avec le Pere Martini, qui dans l'endroit que j'ai cité, et qui est rapporté par le Pere Kirker [sic], parle en ces termes. Je ne sçaurois mieux representer cette racine, qu'en disant qu'elle est presque semblable à notre mandragore, hormis que celle-là est un peu plus petite, quoi qu'elle soit de quelqu'une de ses especes. Pour moi, ajoute-t-il, je ne doute point du tont qu'elle n'ait les mêmes qualitez et une pareille vertu, puisqu'elle lui ressemble si fort, et qu'elles ont toutes deux la même figure." Lafitau a raison dans sa critique qui suit: "Si le Pere Martini a eu raison de l'appeller une espece de mandragore a cause de sa figure, il a eu tort de l'appeller ainsi à cause de ses proprietez. Nos especes de mandragore sont narcotiques, rafraîchissantes, et stupéfiantes. Ces qualitez ne conviennent point du tout au ginseng." Alors Lafitau s'efforce de démuntrer que la mandragore des anciens n'est pas jdentique à notre mandragore d'aujourd'hui. Une autre curiosité de l'opuscule de Lafitau c'est qu'il rapproche le nom iroquois du ginseng canadien, garent oguen (qu'on dit signifier "cuisses, iambes" + "deux choses séparées") au mot chinois traduit par lui "ressemblance de l'homme". Il en conclut que “la même signification n'avoit pû être appliquée au mot Chinois et au mot Iroquois sans une communication d'idées, et par consequent de personnes. Par là je fus confirwe dans l'opinion que j'avois déja, et qui est fondée sur d'autres préjugez que l'Amerique ne faisoit qu'un même continent arec l'Asie, à qui clle s'unit par la Tartarie a nord de la Chine." Tout cela est excusable et intelligible, eu égard à l'état de la science au temps où vivait l'auteur.

1 Je ne crois pas que la traduction de Bretschneider ("has hands, feet, a face and eyes like a man possessed of a god') soit correcte; le mot 融 ne se rapporte qu'à la racine même. 
il y eut un homme à Šñ-tañ ${ }^{2}$ derrière la maison duquel on entendait chaque nuit la roix d'un homme. On le cherchait, mais sans le trouver. En s'écartant un li de la maison, tout ce qu'on aperçut fut une plante de ginseng avec les branches et les feuilles hautes et bien développées. On la déracina et on trouva que la racine avait plus de cinq pieds de long, et que toute sa forme imitait le corps d'un homme. Depuis ce moment les cris cessèrent." 2 A en croire le $P^{*} e i$ wen cai kwain k’ün fan $p^{*} u$ 佩交齊廣荱芳譜 (chap. 93, p. 5 b) il y a encore un texte plus ancien à relever ce trait, le 1 yüan 異苑, attribué à Liu King-šu 劉敬叔 du einquième siècle; mais n'ayant pas à ma disposition une édition de cet ouvrage, je laisse de côté la question chronologique. Lin Kin-šu dit: "Auciennement il $y$ eut un homme qui, en fouillaut le sol, y introduisit sa bêche. Puis il entendit dans la terre des soupirs, et en recherchant le son, obtint de fait un ginseng." 8 Rappelons aussi le fait que les Cbinois se servent de ginseng comme aphrodisiaque.

Ces coincidences étant constatées, les ressemblances entre les traditions de la mandragore et du ginseng sont épuisées, et les différences, au contraire, sont plus nombreuses et plus fondamentales. Le ginseng n'est pas ume plante vénéneuse, elle rétablit la vie et ne donne jamais la mort comme la mandragore. Il n'est pas dangereux ou fatal de recueillir du ginseng qui n'est point devenu objet de magie. Son cri paraît comme un développement logique

- La partie du Śan-si sud-est, toujours fameuse pour son excellent ginseng.

＼cjkstart高祖時上黨有人宅後每夜有人呼聲。求 之不得。去宅一里所但見人參一本。枝葉峻 茂。因掘去之。其根五尺餘。具䯏人狀。呼韾

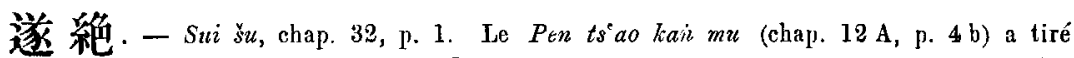

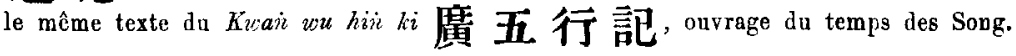

:昔有人掘之始下鑊便聞土中吓吟聲尋 音而取果得人参. 
de sa caractéristique anthropomorphe, et qui plus est, n'envoie pas un homme à la tombe. En effet, los Chinois n'ont rien emprunté de cela aux peuples occidentaux; une telle théorie se heurterait sérieusement contre la chronologie. L'anthropomorphisme et la faculté de parler du ginseng sont d'une date plus ancienne en Chine que les notions analogues de la mandragore à l'ouest; et selon toute apparence, la connaissance de la mandragore n'y est pas arrivée avant l'époque des Soug. Mais s'il est vrai que le ginseng était un objet de commerce de la Chine is la Perse, la question se pose si le cri de la mandragore qui fait son début au moyeu âge n'est pas le résultat direct des contes chinois concernant le ginseng. ${ }^{1}$

Le fait rapporté par Cou $\mathrm{Mi}$ que des racines de mandragore étaient importées en Chine anx temps des Song et effectivement employées n'est pas moins intéressant. Cependant il est frappant que ni Čou K’ü-fei ni Čao Žu-kwa ne paraissent connaitre ce commerce. ${ }^{2}$

Mais Čou K’ü-fei 周去非 nous a laissé une anecdote sur une autre plante apparentée à la mandragore quant à la composition et à l'effet de son poison et qui pour cela ne manque pas de piquer notre curiosité. Aussi nous donnera-t-elle occasion de formuler

1 Il y a d'autres plantes les racines desqquelles sont conçues par les Chinois comme anthropomorphes, par exemple, Plyytolacca acinosa, šai lu 商㫦 (cf. Bretschucider, Chinese Recorder, III, 1871, p. 219; Bot. sin., II, no. 112, 111, no. 131), décrite par le Pie $l u$ avec les mêmes expressions que le ginseng (如人开者有耐) et appeléc aussi ye hu 依 畔 ("criant de nuit"). Mais le Pen ts"ao kai mu ne contient pas de texte qui fasse allusion à la faculté de cricr qu’aurait la racine. Minakata (voir

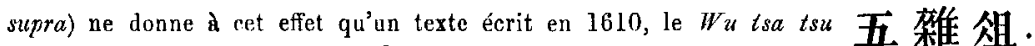

2 La plante lair-tu 狠毒 a été identifiéc avec une mandragore par Bretschneider (Bot. sin., III, no. 132), qui fonda cette opinion sur un dessein japonais, mais Stuart (Chinese Materia Medica, p. 257) regarde cette identification comme douteuse et la description dans les sources chinoises comme insuffisante; à l'avis dı même auteur (p. 58) il est doutenx aussi que le genre Atropa se trouve en Chine. Forbes et Hemsley (.Tournal Linnean Society, XXVI, p. 175) en registrent une Mandragora caulescens au Yun-nau d'après Franchet (Bull. Soc. Bot. de France, XXXII, p, 26). Quoi qu'il en soit, il est certain qu’ancune mandragore n’est connue à la pharmacopée chinoise. 
quelque conclusion à propos du nom mandragore lui-même. Dans son Lin wai tai ta 嶺外代荅, écrit en 1178, l'auteur chinois rapporte ainsi: "La fleur man-t"o-lo de la province de Kwang-si croît partout dans l'état sauvage. Ses feuilles sont larges, les fleurs blauches, et la formation des fruits est comme chez l'aubergine ou la mélongène (Solanum melongena). Elle forme partout des petits piquants, et e'est une plante qui sert de remède aux hommes. ${ }^{1}$ Des voleurs ceuillent la plante, la sèchent et broient. Ils la placent de manière que des hommes la boivent on mangent; et en ce cas ils en deviennent irres. Pendant qu'ils sont daus cet état de torpeur, les brigands enlèvent leurs cassettes et prenuent la fuite. Les hommes au midi de la Chine se servent de ce remède aussi pour les petits enfauts et en amassent de grandes quantités." 2

Le nom man-to-lo 曼陮羅 est conteu dans le Fan yi min $i$ tsi (chap. 8, p. 6) et équivaut au sanskrit mandara, mandāra, mandāraka. ${ }^{3}$ Il est assez étonnant qu'une plante non-cultivée, qui d'après Li Ši-čen croît aussi au nord de la Chine, soit appelée d'un terme sanskrit. Elle n'apparaît pas dans les documents avant l'époque des Song, 4

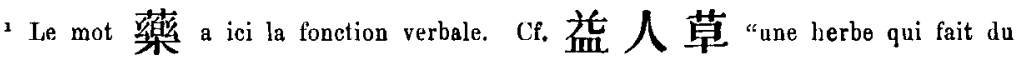

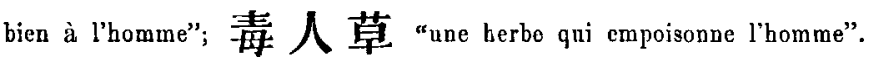

“廣西曼陀羅花徧生原野大葉白花結實 如茄子而徧生小刺乃藥人草也。监賊探乾

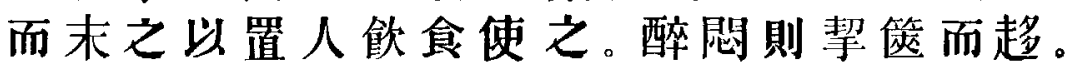
南人或用雼小兒食藥去積甚峻. - Lii wai tai ta, chap. 8, p. 14 b; éd. du Cii pu tsu čai tsiui šu.

3 Voir aussi Eitel, IIandbook of Chinese Buddhism, p. 94.

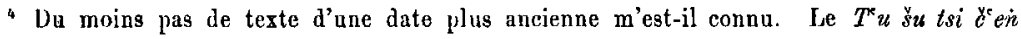
(section botanique, chap. 124), sous le titre $\operatorname{man}-t^{\circ} v-l o$, ne fait que citer la notice du Pen

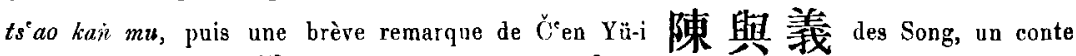
tiré du T'an Yüan 診范 par Yañ I 㛫偣, qui vécut au commencement de l'onzième siècle et collabora au Ts'e fu yüan kwei, et une note très courte du Lo yai hwa mu ki

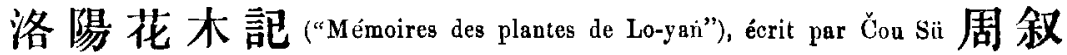
dans la seconde moitié de l'onzième siècle. Ie texte le plus important du Lìn wai tai ta 
et pour cela est suspecte d'avoir été importée de l'Inde, quoique le fait d'une telle importation ne soit pas relevé par les textes. La plante se rapporte au genre Datura, mais il n'est pas certain si c'est l'espèce alba ou stramonium. ${ }^{1}$ C'est une solanée comme la

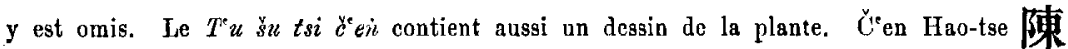

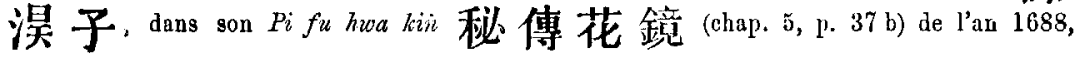
décrit le man-to $t^{\circ} l o$ comme une fleur du nord de la Chine et dit que le nom est sanskrit.

' Stuart, Chinese Materia Medica, p. 145-147. "Le Datura stramonium, ou Pomme éfineuse [anglais thorn-apple], appelé aussi stramoine, endormie, herbe aux sorciers, herbe aux diables, crô̂t communément en France, mais il se rencontre également dans presque toutes les parties du monde, à l'exception de l'Australic; on pense qu'il est originaire de l'Amérique et qu'il s'est propagé de là en Hurope. Cependant on le trouve depuis des siècles, en France, en Grèce, dans la région caucasique, dans la Syrie" (A. Milne Edwards, De la famille des solanacées, p. 87, Paris, 1864). - Les auteurs chinois de l'English and Chinese Standard Dictionary étaient conduits par un sentiment juste, en se servant $\mathrm{du}$ mot man-t'o-lo pour traduiro l'anglais mandrake. S. Couvreur (Dictionnaire francaischinois, p. 369) donne deux termes su-hwa poar Datura: san ma-tse 山府乎 ("chanvre sauvage") et la-pa liwa 涑吹 花 ("leur à trompette"). Le datura est connu aux Cams sous le nom salak et aux Khmers sous le nom slak (Aymonier et Cabaton, Dictionnaire čam-français, p. 481). Notre mot datura est ramené au sanskrit dhattūra par Yule (Ilobsun-Jobson, p. 298); Hindi et Hindustīni dhatüra, persan dätüra s, La mention la plus ancienne du datura qui me soit connue dans la littérature européenne vient de Pierre Belon du Mans, qui dit dans son œuvre Les Olservations de plusieurs singularitez et choses memorables, trounées en Grece, Asie, Iudee, etc., fol. 369 (Anvers, 1555) [ef. T'oung Pao, 1916, p. 362]: "Les Turcs ont des merueilleuses experiences de plusieurs choses, comme pour faire dormir soudainement. Voudroit on chose plus singulière que de trouurer drogue pour faire incontinent dormir qu'elqu'vn qui ne pent reposer? Ils vont chez vn droguiste (car ils n'ont point d'A poticaires) auquel demandent pour demie aspre de la semence de Tatoula. Puis la baillent à celuy qui ne peut dormir. Tatoula n'est autre chose que ce que les drabes appellent $\mathrm{Nux}$ metel, et les Grecs Solanum som. niferum: de laquelle nous en trouuasmes de sauuage en la plaine de Iericho, prés la fon-

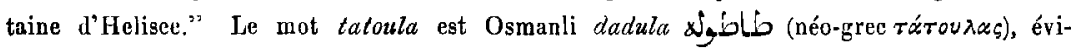
demment dérivé du persan. Cette forme du nom n'est pas notée par Littré qui ue doune que datura et le dérive de l'arabe datora et du persan tatula, en ajoutant "du radical tal, piquer, par allusion à l'enveloppe épineuse du fruit." V i le mot sanskrit, cette étymologie semble être caduque. Christoual Acosta (Tractado delas drogas y medicinas de las Indias Orientales, p. 87, Burgos, 1576) s'exprime ainsi: "Lhamase esta planta en el Malabar, Vnmata [Sanskrit unmatla] caya : en Canarin, Datyro: los Arabes, Nux Methel, y Marana: los Portngueses, Datura, y la Burladora: los Parsios, y Turcos, Datula: los medicos Indianos graduan esta planta fria, euel grado tercero, y seca enel fin del segundo." Acosta donne une gravure de la plante et contribue des observations intéressantes sur son emploi dans l'jnde et l'Espagne. Le mot netel du terme botanique Datura metel, originaire de 
mandragore, et comme toutes les solanées, contient l'alcaloïde daturine ou atropine, $\mathrm{C}_{17} \mathrm{H}_{23} \mathrm{O}_{3}$. L'analogie des contes de Čou $\mathrm{Mi}$ et de Čou K'ü-fei, bien qu’ils se rapportent à des plantes différentes, est due à la composition chimique analogue et à la même action des deux poisons. Encore de nos jours, les Chinois ont employé cette substance funeste pour des buts artificieux. Crawfurd ${ }^{1}$ nous informe que kučubun (le mot soundanais pour Datura ferox) ${ }^{2}$ est donné par les Malais pour produire la plus complète stupeur et "is a powerful engine in the hands of the Chinese for effecting various artifices and tricks in trade." On dit que dans quelques parties de la Chine Datura alba s'emploie pour stupéfier et saisir des poissons. ${ }^{3}$ La désignation propre de la dernière espèce est nao-yain 閙羊; d'autrui identifient ce terme avec Datura metel. Les fleurs, digérées dans le vin, servent d'anesthésique et sont indiquées dans la chorée des enfants; on en fait aussi des lotions contre les éruptions de la face, l'enflure des pieds et la chute du rectum. ${ }^{4}$

l'Inde, est dérivé du sanskrit mätula, peut-être apparenté ì matta et unmatta ("enivré, insensé", et aussi "datura"). Cette espèce est notée par Loureiro (Flora Cochinchinensis, p. 135) pour l'Indochine sous le nom nao yai hwa 幦陽花. Datura ferox ćtait connu à Linné comme une plante chinoise; elle est commune dans la Chine septentrionale (Bretschneider, Early European Researches into the Flora of China, p. 104). Une autre espèce, Datura meteloider, connue en Amérique, est employée par les Indiens Zuñi et Mohave (M. C. Stevenson, Ethnobotany of the Zuñi Indians, Thirteenth Annual Report Bureau of American Ethnology, 1908-09, p. 46; W. E. Safford, Proceedings of the Nineteenth Intcrnat. Congress of Americanists, p. 28, Washington, 1917).

1 History of the Indian Archipelago, I, p. 466.

2 Javanais kačubun, malais kečubun. D'après l'Encyclopadie van Nederlandsch-Indï (II, p. 204) ce mot se rapporter'it au Datura alba.

${ }^{3}$ C. Ford, Flora of Hainan (China Review, XX, p. 161). Le même auteur fait remarquer que cette plante s'appelle à Hoihow mui-twa-lo $\mathrm{PA}$ 山蘿, évidemment une tentative dialectale de reproduire le mot étranger man-to-lo. Forbes et Hemsley (Journal Linnean Society, XXVI, p. 175) disent que Datura alba se trouve dans la Chine méridionale et à Formose, et est cultivée à Peking.

* J. L. Soubeiran et Dabry de Thiersant, La Matière médicale chez les Chinois, p. 190 (Paris, 1874). Dans An Epitome of the Reports of the Medical Officers to the Chinese Imperial Maritime Customs Service, from $18 \% 1$ to 1882, compilé par C. A. Gordon 
E. Perrot et P. Hurrier, ${ }^{1}$ deux pharmaciens français qui ajoutent à la nomenclature chinoise le nom japonais mondarague, donnent les renseignements suivants: "Les grains de ce Datura, irrégulièrement triangulaires et dont la forme a été comparée à celle de l'oreille humaine, sont d'un brun jaunâtre clair, rugueuses, déprimées au centre. Daus l'Inde, elles servent à préparer un extrait et une teinture très estimés comme narcotiques et sédatifs. Les feuilles s'emploient topiquement comme calmantes. Les fleurs, digérées dans le vin, jouissent d'une grande réputation dans l'épilepsie et l'hydropisie." En effet, plusieurs espèces de Datura (fastuosa, metel, et stramonium) croissent dans I'Inde. ${ }^{2}$

C'est dans l'Inde que nous rencontrons aussi le prototype des brigands de Čou K'ü-fei. Nous savons par Garcia da Orta (1563) que les thugs indiens mettaient cette drogue dans la nourriture de leurs victimes, et que l'effet en durait vingt-quatre heures; ceux qui prennent cette médecine perdent leurs sens, rient toujours et sont très généreux, car ils laissent les gens enlever quelconque joaillerie qu'ils choisissent, et ne font que rire ou parlent très peu, et seulement des absurdités. ${ }^{3}$ Les cas d'empoisonnement avec le Datura sont encore très fréquents dans l'Inde. Mais les fripons

(London, 1884), il est dit ( $p .231)$ : "The datura or man-t"o-lo of the Buddhist classics is foreign to China, having, it is said, been introduced from India. When eaten, unconscious laughter is set up, and the person acts as if intoxicated. It may be used as an anæsthetic. It is used in infusion to wash the feet; it is also applied to ulcers of the face, in convulsions of children, and in prolapsus ani." Voir anssi G. A. Stuart. Chinese Materia Medica, p. 145-14i7.

1 Matière médicale et pharmacopée sino-annamites, p. 174 (Paris, 1907).

$=$ W. Ainslee, Materia Indica, I, p. 442-446 (London, 1826); W. Roxburgh, Flora Indica, p. 188; G. Watt, Commercial Products of India, p. 487-489; Flückiger et Hanbury, Pharmacographia, p. 459-463. Datura alba est indigène dans l'Inde; il n'est pas certain si ceci est le cas pour Datura stramonium (A. de Candolle, Géographie botanique, IJ, p. 73l). 'Toutefois cette espèce se trouve dans l'état sauvage à l'Himalaya de Kachmir à Sikkim.

3 C. Markham, Colloquies on the Simples and Drugs of India by Garcia da Orta, p. 175 . 
chinois et indiens qui apparaissent si modernes et civilisés dans leurs méthodes et assez congéniaux à nos chloroform burglars ne peuvent se vanter d'une grande originalité. La ruse est vieille, hors que les anciens préparaient l'extrait non du Datura, mais de la mandragore; e'est toute la même chose. Frontin qui vécut sous les règnes de Vespasien et de ses fils, et mourut dans les premières années du règne de Trajan, raconte dans ses Stratagèmes l'anecdote suivante: "Maharbal, envoyé par Carthage contre les Africains révoltés, sachant cette nation très-portée pour le vin, en fit mêler une grande quantité arec de la mandragore, substance qui tient le milieu entre un poison et un soporifique; puis, après une escarmouche, il se retira. Vers le milieu de la nuit il fit semblant de prendre la fuite, laissant quelque bagage et tout le vin empoisonné. L'ennemi se jeta dans le camp; et là, dans la joie de la victoire, ayant bu avec excès de cette mixtion, tandis qu'ils étaient étendus par terre comme des corps morts, Maharbal revint sur ses pas, et en fit un grand massacre". I Polyen (Polyainos) de la Macédoine, qui récut à Rome sous les règnes de Marc Aurel et L. Verus, dit dans son Strategika (VIII, chap. XXIII, 1) que le jeune César, en voyage pour l'Orient, tomba dans les mains de pirates ciliciéns pas loin du cap Malea. Il fit venir la rauçon demandée de Milet et au même temps un pot rempli d'épées et une quantité de vin empoisonné arec de la mandragore. Il en régala les pirates et ordonna qu'ils fussent massacrés dans leur assoupissenent. Dans un autre passage du même ouvrage $(V$, chap. $X, 1)$ Polyen rapporte un conte sem-

- Maharbal, missus a Carthaginiensibus adversus Afros rebellantes, quum sciret, gentem avidam esse vini, magnum eius modum mandragora permiscuit, cuius inter venenum ac soporem media vis est. Tunc, proelio levi commisso, ex industria cessit: nocte deinde intempesta, relictis intra castra quibusdam sarcinis, et onui vino infecto, fugam simulavit: quumque barbari occupatis castris, in gaudium effusi, medicatum avide merum hausissent, et in modum defunctorum strati jacerent, reversus aut cepit eos, ant trucidavit (Strategematicon II, chap. V, 12). J'ai reproduit la traduction de Th. Baudement dans l'édition d'Amien Marcellin, Iornandès, l'rontin, etc., par M. Nisard, p. 536 (Paris, 185́l). 
blable à celui de Frontin à propos du général carthaginien Himilco.

Mais retournons à l'Inde. La connaissance du dhattūra y remonte à une époque reculée, car la plante est plusieurs fois mentionnée par Suçruta. Je dois à l'obligeance du Dr. A. F. R. Hoernle d'Oxford les renseignements suivants:

"There are the following references to dhattura in the text-book of Suçruta:-

1. Cikitsāsthäna, ch. XVII, verse 35, p. 435 (Jīvĩnanda, 5th el.), where pounded seeds of dhattūra and other drugs (madana, kodrata, etc.) are recommended in the treatment of a sinus $(w a \bar{a}(\bar{i})$.

2. Kalpasthāna, ch. VI, verse 49, p. 589, roots (or, accordiug to others, seeds) of dhattura, made up, with other drugs, into a paste, is recommended as a çirovirecana ('clearing of the head') in the case of hydrophobia.

3. Ibidem, verses 51, 52, root of dhattura, made, with other drugs, into a paste, wrapped in leaves of unmattaka (synonyme of dhattüra), and baked into a cake (apupaka), is recommended in the case of bite by a rabid dog.

"In the text-book of Caraka, dhattūra does not occur at all. For the occurrence of dhattinra in medical text-books and later authors, such as Cakrapāṇidatta, c. A.D. 1060, and Bhāva Miçra (Bhävaprakäça), 16. cent. A.D., see U. C. Dutt's Materia Medica, pp. 207--8.

"The Sanskrit word is spelled variously dhattiura, dluttūra, dhüsstüra, etc.

"You identify dhattūra with Datura stramonium; but, as you know, our botanical books mention other varieties also. Sanskrit medical text-books distinguish two varieties, black, or rather dark, flowered, and white flowered (see Mat. Med., p. 202); and they bave also two uames, dhattūra and unmatta (or unmattaka). The former is expressly called krgnna-pusspa, 'dark-flowered' (see Rāja Nighantu, 10. varga, p. 135), and appears to be the one usually intended to 
be used. But unmatta seems to be the proper name of the white variety, indicated by a prescription of Cakrapānidatta (Mat. Med., p. 207, note 2), where the name çvetonmatta, or 'white dhattūra' occurs. D. stramonium has white flowers, while $D$. metel and D. fastuosa have darker flowers. Accordingly the Sanskrit name dhatturra would seem to refer to the latter two varieties, while unmatla would seem to indicate the rariety D. stramonium." 1

1 Le Dr. T. Tanaka an Burean of Plant Industry, Department of Agriculture, Washington, a eu l'extrême obligeance de traduire pour moi les renseignements suivants sur Mandara-lwwa (ou Mandara-ge selon la prononciation bouddhiste) 曼陇羅化,

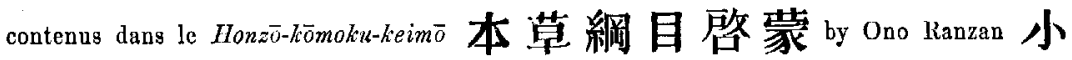
野蘭山 (revue par lguěi Boši 井口热之, 1847, chap. 13, p. 28-29):

“.7apanese Nomenclature:

In Provinces:

Cósen-asagao (Korean morning glory).

Yama-nasubi (mountain egg-plant).

Namban-asagao (morning glory of the Sonthern Barbarians).

Hari-nasubi (spiny egg-plant).

IV̄-nasubi (Chinese egg-plant).

1 yo.

Gelkwa-korosi (scrgeon killer).

Iyo.

Cekwa-dausi (sergeon thrower).

Sanuki.

T'enjiku-nasubi (Indian egg-plant).

Hōki, Iwami, Iyo.

lga-nasubi (prickly egg-plant).

Awa.

Giba-sō (meaning uncertajn).

Awa.

Camera-so (meaning uncertain).

Buzen.

Kičigai-nasubi (insane eggr-plant).

Edo (Tōkyō).

Awisu (meaning uncertain).

Iwami.

1ga-nasu (prickly egg-plant).

Bingo.

$K i$-asagao (tree [or yellow ?] morning glory).

Nagato.

Cósen-tabako (Korcan tobaceo).

Simoosa.

Trotōmi.

T'o-asagao (Chinese morning glory).

Baramon-so (herb of the Baramon 浓羅朋, that is, Brishmana).

Chinese synonymes :
佛花
Fu hwa ('Buddhu's flower'). 和幼新書
偭茄
Tien kie.
香山縣志
煀陀羅草 Men to to ts'ao.
名山勝概
天茄輛陀花 Tien k'ie mi to howa.
花曆百詠

"Spontaneous in the provinces Hoki, Buzen, and Suwo, but not grown in the prefectures 
Ainsi l'histoire du genre Datara dans l'Inde est assez claire. Quant au mot mandara, nous avons noté que les Chinois et les Japonais le rapportent exclusivement au datura. En consultant le dictionnaire sanskrit de Boehtlingk, nous trouvons que mandara, mandāra ou mandāraka signifient en premier lieu Erythrina indica, ${ }^{1}$ l'arbre de corail, un des cinq arbres du ciel d'Indra, appelé aussi parijäta, puis une variété blanche de Calotropis gigantea, et enfin la pomme épineuse, c'est-à-dire le genre Datura. A l'égard de ces identifications, il est évident que le terme mandara, quand il est mentionné dans les textes bouddhistes chinois où la plante tombe des cieux comme une pluie au temps où le Bouddha prêche la loi, est l'Frythrina à l'exclusion du Datura. ${ }^{2}$ D'autre part, l'usage du mot mandara chez Cou K'ü-fei à l'époque des Song prouve assez bien que dans l'Inde aussi mandara servait d'expression pour le Datura.

near Kyoto. The seed is planted in the spring. The form of the leaves is like that of the egg-plant (Solanum melongena), without spines, green, and alternate. The plant is 2-3 feet high, the way of branching being also similar to that of the egg-plant; it blooms in the summer and autumn. Flower standing in axil of leaves, white, resembling the blossom of the morning glory (Pharbitis nil) with elongated tube and united petal. There are five edges on the outer margin of a flower, graduslly narrowed into a tube, about 3 sun (1 sun = 1.193 inch) long. Fruit, about 1 sun long, is round and sping, hence the name hari-nasubi is derived; it contains flat, brownish-black seeds. The plant dies out in the autumn, and no part of it thrives until next year.

"If one happens to eat the flower and leaves by mistake, a nervous condition of the nature of insanity will be the consequence, but with the removal of the virus which caused the effect, this condition is gradually overcome, the result being a complete cure without leaving any mental disorder."

1 W. Roxburgh, Flora Indica, p. 541.

2 Contrairement à ce que Stuart (Chinese Materia Medica, p. 145) dit à ce sujet. C'est d'ailleurs Li Ši-čen lui-même qui est responşable pour cette erreur, en introduisant sa notice sur le man-to-lo arec les mots: "ll est dit dans le Fa hwa kid (Saddharmapaṇigrika-sutra) qu'au temps où le Buddha prêcha la loi, le ciel fit pleuvoir des fleurs de man-t'0-lo." Il ne savait pas que mandara se rapporte dans l'Inde à des plantes difrérentes. 
Voici enfin une question que je me demande et que je voudrais proposer aux étudiants des langues indo-européennes pour leur considération: serait-il possible que le terme sanskrit mandāraka et le terme gréco-latin mandragora(s) soient anciennement apparentés et descendent d'une racine commune? L'accord est éclatant, et si c'est un accident, l'accident serait extraordinaire. 